\title{
Las ilustraciones realizadas por Alicia Bustamante Vernal en carátulas de libros y en revistas (1938-1962)
}

\section{Illustrations Made by Alicia Bustamamte Vermall on Book Covers and Magazines (1938-1962)}

\author{
Georgina Antonieta Verde Márquez \\ Universidad Nacional Mayor de San Marcos, Lima, Perú \\ Contacto: gverdem@unmsm.edu.pe \\ https://orcid.org/0000-0003-2313-1698
}

\section{RESUMEN}

El presente artículo tiene por objetivo analizar las ilustraciones en carátulas de libros y en revistas elaboradas por Alicia Bustamante Vernal desde el año 1938 hasta 1962. Ello debido a que se desconoce el significado del trabajo gráfico de esta notable artista, porque esa parte de su vida todavía no ha sido materia de investigación. La metodología propuesta consiste en la búsqueda de la información sobre el tema, para luego proceder a la redacción de la descripción y análisis de los diseños ubicados. Ella ilustró las obras del escritor José María Arguedas: Canto kechwa (1938), Yawar fiesta (1941) y La agonía de Rasu Niti (1962). Además, participó con tres dibujos en "Noticia histórica del actual edificio de la Universidad de San Marcos", que corresponde al primer capítulo del libro El actual edificio de la Universidad Nacional Mayor de San Marcos (1951) de Daniel Valcárcel. Asimismo, diseñó las viñetas del número 9 de la revista 3 (1941), y los dibujos para los relatos de Arguedas titulados El zumbayllu (1951) y Orovilca (1954) publicados en la revista Letras Peruanas. Las ilustraciones de Bustamante Vernal representan variados aspectos de la cultura peruana, entre ellos a la flora, a la fauna, el arte popular, costumbres y la arquitectura. La creatividad de la artista plasmada en cada uno de sus diseños permite tener mayores referencias sobre su obra. Sus ilustraciones están ligadas a un pormenorizado estudio de las tradiciones del Perú, en ellas destacó su gran respeto por la diversidad cultural del país.

Palabras clave: Alicia Bustamante Vernal; Mujer artista; llustraciones; Perú; José María Arguedas.

\section{ABSTRAC'T}

The objetive of this article is to analyze the illustrations on book covers and in magazines produced by Alicia Bustamante Vernal from 1938 to 1962. Because the meaning of the graphic work by the notable artist is unknown, because that part of her life is still it has not been the subject of research. The proposed methodology consist of searching for information on the subject, and then proceeding to the writing of the description and analysis of the located designs. She illustrated the works of the writer José María Arguedas: Canto kechwa (1938), Yawar fiesta (1941), and La agonía de Rasu Ñiti (1962). In addition, she participated with three drawings in "Noticia histórica del actual edificio de la Universidad de San Marcos", which corresponds to the first chapter of the book El actual edificio de la Universidad Nacional Mayor de San Marcos (1951) by Daniel Valcárcel. She also designed the vignettes for number 9 of the magazine 3 (1941) and the drawings for the texts by Arguedas entitled El zumbayllu (1951) and Orovilca (1954) published in the magazine Letras Peruanas. Bustamante Vernal's illustrations represent various aspects of Peruvian culture, including flora, fauna, popular art, customs, and architecture. The creativity of the artist embodied in each of her designs allows for greater references to her work. Her illustrations are linked to a detailed study of the traditions of Peru, in them she highlighted her great respect for the cultural diversity of the country.

Keywords: Alicia Bustamante Vernal; Women artists; Illustrations; Peru; José María Arguedas. 


\section{Introducción}

La pintora peruana Alicia Bustamante Vernal nació en la ciudad de Lima el 14 de mayo de 1905 y falleció el 27 de diciembre de 1968 (Milla, 1996, p. 184). Hasta la actualidad, ella es recordada debido a que fundó junto a su hermana Celia, en 1936, la peña Pancho Fierro. Aquel lugar fue considerado "el más importante escenario de tertulia y reflexión en torno a los acontecimientos políticos, literarios y artísticos del Perú y del mundo" (Carpio e Yllia, 2006, p. 45). Además, fue una reconocida coleccionista de arte popular.

Bustamante Vernal inició sus estudios en la Escuela Nacional de Bellas Artes (ENBA) en 1920 (Escuela Nacional de Bellas Artes, 1922, p. 27). Formó parte de la segunda promoción de alumnos inscritos en la institución. La ENBA fue creada durante el gobierno de José Pardo en 1918, pero inició sus actividades académicas a partir de 1919. $\mathrm{El}$ primer director de la ENBA fue el pintor Daniel Hernández. La institución se encargó de formar a los artistas peruanos más destacados del siglo XX. Bustamante Vernal, en su alma máter, fue alumna de José Sabogal, líder del grupo indigenista; además conoció allí a los otros integrantes de esta corriente cultural: Julia Codesido, Camilo Blas, Teresa Carvallo y Enrique Camino Brent. Sabogal fue el propulsor del indigenismo en la pintura y "viajó a diferentes partes del país" para captar "la geografía y costumbres propias de cada zona"; también retrató a la "población andina, sus costumbres y paisajes", de la misma manera que a "los habitantes de la selva y de la costa" (Verde Márquez, 2016, p. 57). Si bien tenían mucho en común, "cada artista que formó parte de esta agrupación poseía particularidades individuales en la manera de interpretar y expresarse, con temas referidos al Perú" (Verde Márquez, 2015a, p. 18).

El movimiento indigenista se inició en la década del veinte durante el gobierno de Augusto B. Leguía (1919-1930), cuando los intelectuales peruanos planteaban revertir el abandono sufrido por las comunidades andinas (Verde Márquez, 2015a, p. 17). Su relación con ellos se reflejó en dos cuadros: el primero del año 1924, cuando su amistad con Codesido "quedó plasmada en el lienzo" en el Retrato de Alicia Bustamante; el segundo, en 1931, de la mano de su maestro Sabogal en la obra Alicia y Celia Bustamante (Verde Márquez, 2016, p. 58). La relación amical se mantuvo con el paso del tiempo, porque interactuaban en la peña Pancho Fierro. En aquel local se organizó una exposición titulada "Galería de Pintura Peruana", en 1938, que contó con la participación de Bustamante Vernal y los integrantes del grupo indigenista (Falcón, 1989, p. 15). Por tal razón no era extraño el trato cordial entre ellos. De la misma manera, la artista se reencontraría con ellos, en 1946, para trabajar en el Instituto de Arte Peruano en la recopilación del arte popular de las distintas regiones del país para formar la colección de aquel recinto (Wiesse, 1957, p. 59).

En el campo artístico Bustamante Vernal ganó el Primer Premio de Pintura por la ciudad de Lima en 1932 (Tauro del Pino, 1966, p. 218). Al finalizar sus estudios, en la década del treinta, se dedicó a la docencia de "artes plásticas en varios colegios de Lima" (Milla, 1996, p. 184). Su labor de pintora prosiguió en la década del cuarenta y del cincuenta. En 1944, su primera muestra individual tuvo lugar en el Instituto Cultural Peruano Norteamericano, donde abordó el "paisaje de la zona Sur del Perú y de la altiplanicie boliviana" (Fuentes, 1944, p. 3). La segunda exposición individual fue realizada en $1955^{1}$, en el Instituto de Arte Contemporáneo, y reunió sus "últimos trabajos pictóricos" (Exposición en el IAC, 1955, p. 17). La difusión de ambas exposiciones en la prensa daba cuenta de la buena acogida que tenía su trabajo en el medio cultural de aquellos años.

En 1939, su hermana Celia se casó con José María Arguedas (1911-1969). Durante el tiempo que tuvo vigencia el matrimonio, su cuñado Arguedas le encargó a Bustamante Vernal la ilustración de varios de sus textos. El vínculo familiar entre ambos finalizó en 1965, tras el divorcio de la pareja.

Debido a que se trata de la labor de una mujer artista, es necesario tener un breve panorama de la situación de los derechos ciudadanos femeninos. El tema se puso en debate gracias a la organización de María Jesús Alvarado llamada Evolución femenina (1914) (Gonzáles, 2005, p. 28). La reivindicación de los derechos de las mujeres fue continuada por Zoila Aurora Cáceres, creadora de Feminismo Peruano (1924), que defendía la "lucha por el sufragio" (Gonzáles, 2005, p. 31). Después de varias décadas, el presidente Manuel Odría promulgó la Ley n. ${ }^{\circ} 12391$ de 1955 que les otorgó "el derecho al sufragio" (Gonzáles, 2005, p. 38). 
El análisis de la obra plástica de Bustamante Vernal toma como punto de partida el año de 1938, fecha en que se publicó Canto kechwa, período en el cual gobernaba Óscar Benavides (1933-1939). En la década del treinta, la influencia del movimiento indigenista en la pintura estaba en pleno apogeo, pero surgía el cuestionamiento al grupo porque se exigía libertad de elección en lo referido a la temática dentro de la pintura nacional. Se consideraba que los pintores indigenistas desde las aulas de la ENBA, y Sabogal como director (1932-1943), decidían los temas a tratar. Por ello, en 1937 se realizó el Primer Salón de los Independientes, que reunió a los opositores a la propuesta de Sabogal (Castrillón, 2001, pp. 20-24). Tras la salida de Sabogal de la ENBA en 1943, la dirección de la institución quedaría dos años más tarde en poder de Ricardo Grau (1907-1970), cuya obra pictórica fue aceptada por su destreza en el uso del color. La presente investigación tiene como fecha límite el año de 1962, que coincide con el último período de mandato del presidente Manuel Prado Ugarteche (1956-1962). En esta etapa, la influencia del indigenismo en la pintura había quedado de lado. En el medio cultural, el arte abstracto era aceptado de manera oficial porque en el Museo de Arte de Lima, en 1958, se presentó la muestra titulada Primer Salón de Arte Abstracto (Salazar Bondy, 1958, p. 8).

Después de plantear el contexto, se debe precisar que el objetivo del presente artículo busca determinar las ilustraciones realizadas por Alicia Bustamante Vernal en carátulas de libros y en revistas desde el año de 1938 hasta 1962. No existen investigaciones dentro de la historia del arte peruano sobre las ilustraciones de esta artista. El punto de atención sobre ella siempre ha estado referido a su papel de coleccionista de arte popular, aunque su desarrollo en el campo de la ilustración abarcó alrededor de dos décadas. Debido al escaso interés que ha suscitado el análisis de su obra plástica, en el presente trabajo se indaga por el significado de sus ilustraciones en carátulas de libros y en revistas en poco más de dos décadas. Los nombres científicos de las plantas y animales plasmados en los diseños se desconocen, porque no han sido materia de análisis en ninguna publicación académica. De tal manera, esta investigación contribuye a desvelar los datos que han permanecido ocultos en los diseños por más de sesenta años. Las obras de Arguedas tituladas, Canto kechwa (1938), Kawar fiesta (1941), La agonía de Rasu Niti (1962), El zumbayllu (1951) y Orovilca (1954) han sido analizadas por la crítica literaria, aunque nunca se recurrió a la botánica para identificar a la flora presente en los diseños que acompañan a los textos mencionados.

Se plantea que Alicia Bustamante Vernal trabajó con los intelectuales interesados en representar a la naturaleza y tradiciones del Perú, en el período bajo la influencia del indigenismo pictórico, y ella en sus ilustraciones utilizó la flora, fauna y tradiciones propias de la cultura andina para sintetizar de esa manera los conocimientos que adquirió de tales poblaciones en su constante interrelación con ellos. En la presente investigación planteo el acápite llamado "La participación de Alicia Bustamante Vernal en la ilustración de carátulas de libros y en revistas", en el cual se describen cada uno de los diseños hallados y se interpreta sus significados. Se tratan las ilustraciones contenidas en los siguientes textos: Canto kechwa (1938), Yawar fiesta (1941), La agonía de Rasu Ñiti (1962), "Noticia histórica del actual edificio de la Universidad de San Marcos", 3 (1941), El zumbayllu (1951) y Orovilca (1954).

Se analizan las ilustraciones sobre la base del método propuesto por Óscar Morriña en su texto Fundamentos de la forma (1982). El autor plantea tres etapas para el análisis de una obra de arte. La primera parte debe enfatizar en la descripción de los principales datos de la obra. La segunda evalúa los elementos plásticos de la composición que incluye una detallada descripción de la obra. La tercera recopila toda la información para valorar y explicar el significado de la obra de arte como producto de una época determinada. En mi tesis titulada "La representación femenina en la obra de Julia Codesido (1920-1938)" (2016) analicé las ilustraciones de una pintora y puse en práctica el referido método. Ese texto sirvió de guía para la presente investigación.

Después de elaborar el esquema de la investigación, recurrí a la búsqueda de las fuentes en la Biblioteca Central Pedro Zulen, la Biblioteca de la Facultad de Ciencias Sociales, la Biblioteca de la Facultad de Letras y Ciencias Humanas de la Universidad Nacional Mayor de San Marcos, Biblioteca Nacional del Perú y en el Centro de Estudios Literarios Antonio Cornejo Polar. La última 
etapa consistió en procesar la información obtenida y redactar el artículo.

\section{La participación de Alicia Bustamante Vernal en la ilustración de carátulas de libros y en revistas}

La ilustración de carátulas de libros y de revistas tuvo una buena acogida en el país durante la década de 1920. Con el paso del tiempo, esta interrelación entre artistas y escritores se mantuvo vigente. Un ejemplo de ello fue el trabajo gráfico de Julia Codesido en el libro Siete ensayos de interpretación de la realidad peruana (1928) de José Carlos Mariátegui, y en la revista 3 (1941) (Verde Márquez, 2016, p. 59).

En el presente acápite se procede al análisis de las ilustraciones de Bustamante Vernal plasmadas en libros y revistas para conocer el significado de cada una de ellas. Además, se identifica a las personas con quienes tuvo algún vínculo laboral, la información obtenida servirá para conocer en cierta medida el "ambiente cultural de la época" en que ella vivió (Verde Márquez, 2016, p. 13).

\subsection{Canto kechwa (1938)}

Arguedas (1938) recopiló veintiún canciones de la tradición andina para su libro Canto kechwa. El texto está escrito en dos idiomas: el quechua y su traducción al español. Bustamante Vernal realizó la ilustración de la carátula y los "dibujos" del libro (Arguedas, 1938, p. 3).

Figura 1. Detalle de la ilustración de Alicia Bustamante en Canto kechwa (Arguedas, 1938, p. 1). Foto: Georgina Verde Márquez, 2015. ${ }^{2}$

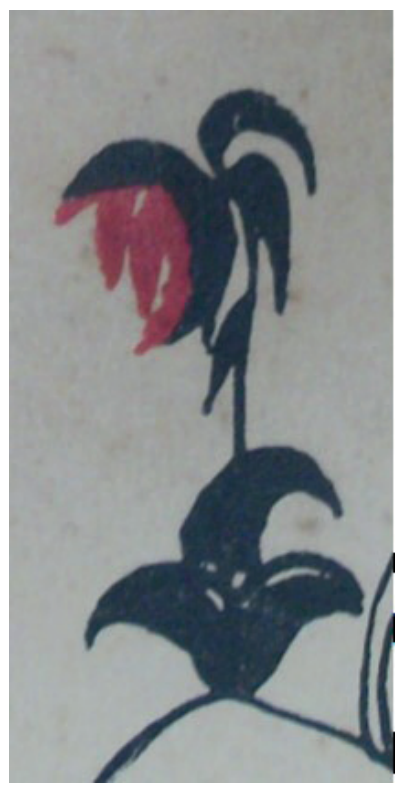

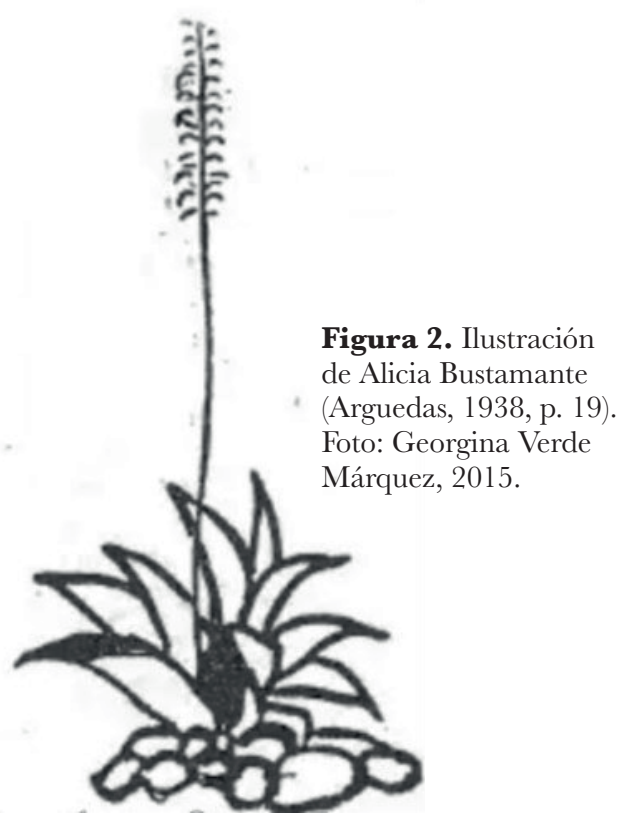

La tapa del libro de color marrón claro sirve de fondo a la carátula, la cual tiene dos secciones, una superior con la tipografía negra en minúsculas dispuesta en cinco renglones, y la inferior que lleva el dibujo. En el primer renglón está escrito el nombre del autor "josé maría arguedas" [sic]; en el segundo, el título del libro "canto kechwa"; y del tercero al sexto figura el título del encabezado del prólogo "con un ensayo sobre la capacidad de creación artística del pueblo indio y mestizo" (Arguedas, 1938, p. 1). El dibujo delineado en negro está compuesto de dos camélidos sudamericanos que se miran frente a frente, ambos de perfil, separados entre ellos por una planta con su inflorescencia roja, la cual crece sobre un montículo (Arguedas, 1938).

Además, la pintora compaginó el libro con cuatro ilustraciones. La primera viñeta es de una planta, que está en la página de presentación del título "canto kechwa" (Arguedas, 1938, p. 19). La segunda viñeta corresponde a las siluetas de dos peces, uno al lado del otro, para la canción "El agua negra...", donde se menciona que la vida de los peces cambia con la llegada de los "repuntes" (Arguedas, 1938, p. 39). La tercera viñeta es de un ave que sostiene una hoja con su pico mientras se posa sobre una rama, para la canción "Llorabas solo patito", al cual se le propone hacer un "nido" (Arguedas, 1938, p. 41). La cuarta es de dos aves distanciadas una de la otra, ambas posadas sobre una rama horizontal, en la canción "Como dos palomas...", donde un hombre lamenta regresar a su pueblo sin la "mujer" con la que había partido (Arguedas, 1938, p. 51). 
En el diseño de la carátula se identificó a la naturaleza, primero a la fauna a través de la vicuña (Vicugna vicugna mensalis), especie de gran importancia económica por la fineza de su pelo. Se determinó las características físicas del animal por la forma de su cuerpo, que lo diferencia de las otras especies de su género. La vicuña conforma el grupo de los cuatro camélidos sudamericanos que existen en el Perú junto a la alpaca, la llama y el guanaco. En el arte peruano se le representó en las cerámicas escultóricas de la cultura Wari (600 d. C.-1000 d. C.) que pertenece al período llamado Horizonte Medio según la clasificación de John Rowe. Durante el virreinato se incorporó a la vicuña en el volumen II de la obra Trujillo del Perú (1782-1785) del obispo Baltasar Jaime Martínez Compañón y Bujanda (1735-1797). La vicuña fue dibujada en la "Estampa 113" titulada "Chaco de vicuñas” (Martínez Compañón, 1998, s/p.). En la república, la vicuña fue uno de los símbolos presentes en el escudo de 1825. El diseño estuvo a cargo de José Gregorio Paredes, y Francisco Javier Cortés realizó el dibujo (Fernández, 1972, p. 484). La elección de la vicuña en el escudo, debido a su comportamiento especial, se explicó de la siguiente manera:

La vicuña, sagrada por su finura y su independencia, es una muestra de tranquilo valor. De los machos que entre los animales llevan a sus hembras en tropilla, es el único que no corre más que ellas para salvarse en los peligros, sino que las hace fugar mientras queda solo, dando cara a la muerte. Ni bestia servil ni animal de doméstico provecho, jamás cautivo, es el fino y valiente señor de nuestras soledades. (Fernández, 1972, p. 485)

Por lo tanto, la imagen de la vicuña en el arte se ha mantenido vigente desde el período del Perú Antiguo, el virreinato, la república, hasta la actualidad.

Cabe agregar que la vicuña es un animal silvestre de pelaje marrón rojizo, con el pecho y mentón blanco, que habita en la puna. Un lugar creado para su conservación y protección se ubica en la reserva nacional de Pampas Galeras, en el departamento de Ayacucho, donde la superficie y los abrevaderos de agua permiten su supervivencia. Los pobladores de la zona andina con facilidad las reconocen a simple vista, en cambio un forastero confunde con facilidad a los cuatro camélidos sudamericanos ya citados.
En el texto se menciona a la vicuña en la canción “¡Ay, flor morada!”, donde una mujer se lamenta del comportamiento de su enamorado y refiere que mejor hubiera "amado a la vicuñita que llora en la orilla de las lagunas sobre las cumbres y en las lomadas" (Ortiz, 1997, p. 356). En la canción, la vicuña forma parte del paisaje; además, habita cerca de las fuentes de agua para sobrevivir. Debido a la importancia de la vicuña para los habitantes andinos era ineludible su elección para la carátula.

También la flora está presente por medio de una planta andina que por la forma de sus hojas e inflorescencias laxas corresponde a la quinua (véase imagen 1), familia Chenopodiaceae y género Chenopodium quinoa. De la misma manera, en las cuatro viñetas contenidas en el interior del libro también se alude a la naturaleza, al igual que en las canciones. La flora está representada por el maguey (Furcraea andina), (véase imagen 2), especie silvestre de la región quechua, que es una planta fácil de reconocer en el paisaje andino. La fauna acuática está presente por medio de los peces que viven en los ríos de la sierra. El diseño se relaciona con el tema de la canción, pues la vida de los peces en el agua cristalina cambia definitivamente cuando llega el "repunte" con agua turbia. A su vez, las aves hacen referencia a las relaciones sentimentales de los enamorados. Primero un ave sobre una rama se prepara para la construcción de un nido e invita al "patito" de la canción para realizar tal tarea (Arguedas, 1938, p. 41), entonces significaría la unión de una pareja. Según Alejandro Ortiz ${ }^{3}$ (1997), cuando un "pato" llora "hiere el corazón del amante solitario" (p. 363). Luego dos aves están separadas en una rama horizontal ondulada, lo que señala el fin de la relación entre un par de enamorados, porque en la canción el hombre regresa solo a su pueblo (Arguedas, 1938, p. 51).

Otro dato interesante sobre la carátula de Canto kechwa es su mención en la Enciclopedia del arte en América, Biografías III (1968) de Vicente Gesualdo, donde se indica que Bustamante Vernal "ilustró" el libro de Arguedas (Gesualdo, 1968, s/p). También, en el artículo "Alicia y Celia Bustamante, la Peña Pancho Fierro y el Arte Popular" (2006) de Kelly Carpio y María Eugenia Yllia, se comenta que "Canto kechwua" [sic], en su primera edición de 1938 "llevaba ilustraciones de Alicia Bustamante y Wilfredo Navarrete" (Carpio e Yllia, 2006, p. 48). Sin embargo, esa afirmación es incorrecta porque 
en la primera edición de 1938 solo Bustamante Vernal ilustró la carátula y también las viñetas. La información del artículo mencionado corresponde a la versión de Editorial Horizonte publicada en 1989, que difiere de la primera edición de 1938.

En la presente investigación, las ilustraciones de la carátula e interior del libro Canto kechwa (1938) corresponden a la primera colaboración de Bustamante Vernal con el escritor; la interacción entre ellos se debía a que él "compartía los mismos intereses que ambas hermanas, pues su trabajo intelectual estaba dedicado a revalorar la cultura peruana en diversos aspectos" (Verde Márquez, 2016, p. 58).

Se puede inferir que la vicuña en la escena "fue creada para resaltar la persistencia de una cultura rica en tradiciones, la andina, vigente en sus depositarios los peruanos" con el fin de "indicar que existe permanencia y continuidad de costumbres" (Verde Márquez, 2016, p. 107).

\subsection{Yawar fiesta (1941)}

En la presente investigación se considera la primera edición de la novela, publicada en 1941 por la Compañía de Impresiones y Publicidad (C.I.P.) con la ilustración de Bustamante Vernal en la portada. Sin embargo, hubo una segunda edición con modificaciones que publicó en julio de 1958 la Editorial Juan Mejía Baca (Rodríguez, 1984, p. 9).

El libro Yawar fiesta, de Arguedas, contiene muchas alocuciones en quechua. Cornejo Polar (1973) refiere que Yawar fiesta "afirma la existencia de dos mundos distintos y opuestos, en aguda contienda" (p. 93). El tema central trata sobre los preparativos para el "turupukllay" en Puquio, que se realizaba el 28 de julio en el "yawar fiesta" (Arguedas, 1958, p. 149). Una de las divinidades de los pobladores de Puquio se denominaba el "auki K'arwarasu"6 que era el "padre de todas las tierras de Lucanas", a quien le pedían protección para capturar al toro llamado "Misitu"; el animal era el centro de atención en la ceremonia (Arguedas, 1958, p. 105). De esa manera, las creencias religiosas de los pobladores formaban una parte fundamental de sus fiestas tradicionales. Sin embargo, el subprefecto del pueblo recibió "una circular de la Dirección de Gobierno" que prohibía la corrida, por ser considerada una actividad peligrosa para la integridad física de los pobladores (Arguedas, 1958, p. 42). En la novela, la fiesta había cambiado respecto al pasado, veinte años atrás, porque ya no se "amarraba un cóndor al lomo del toro para ponerlo más furioso por la picadura", dicha acción fue reemplazada por la dinamita (Arguedas, 1958, p. 114).

Bustamante Vernal ilustró la "carátula" del libro Yawar fiesta (Arguedas, 1941). La tapa del libro de color marrón claro tiene una tipografía negra. En el margen superior lleva el título "Yawar fiesta", y en el margen inferior el nombre del escritor "José maría Arguedas" [sic] (Arguedas, 1941). En la parte central figura una planta (véase imagen 3) compuesta por tres inflorescencias, dos laterales de perfil y una en medio, del suelo crecen cinco hojas lanceoladas verticales acomodadas en forma de roseta. Detrás de la planta a manera de fondo ondea una tela cuadrangular de color marrón oscuro. Además, se percibe en la contracarátula el dibujo de la misma planta de la portada (Arguedas, 1941), que está conformada por dos inflorescencias de perfil entrecruzadas con sus hojas lanceoladas dispuestas en forma de roseta.

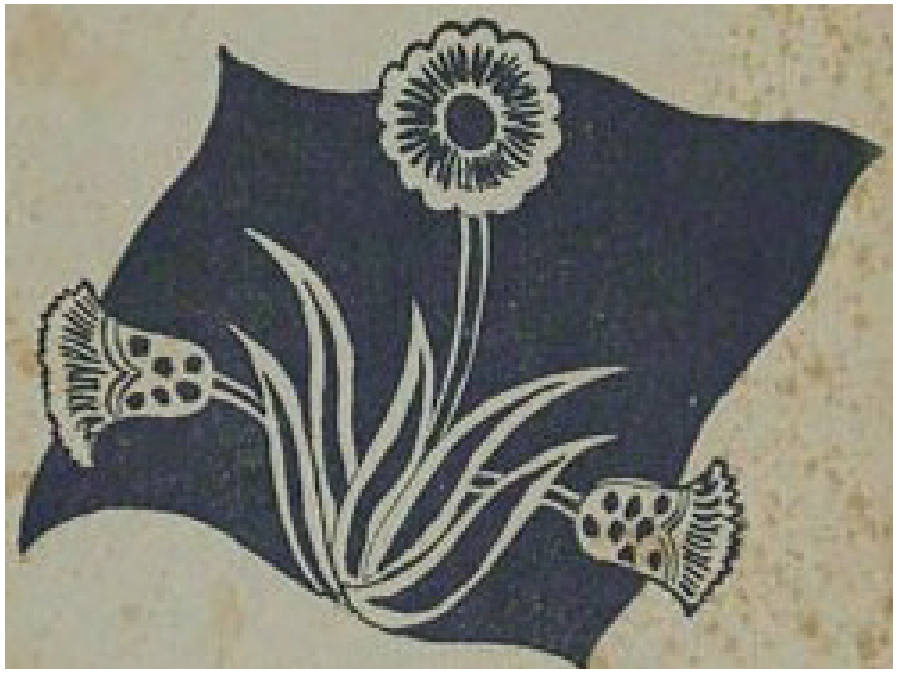

Figura 3. Ilustración de Alicia Bustamante (Arguedas, 1941, p. 1). Foto: Biblioteca Nacional del Perú, 2020.

Luego de la descripción de los diseños es necesario identificar el nombre científico de la planta y explicar por qué Bustamante Vernal la escogió. Así, después de varios infructuosos años de investigación, se logró determinar - por su porte arrosetado, la inflorescencia llena de pelos pubescentes y flores blancas - que la planta pertenece a la familia 
Asteraceae y al género Werneria nubígenea. En el ámbito académico no fue fácil dar con el nombre de la especie, incluso para los expertos en el tema hasta de la propia zona de origen de la planta. El dato está restringido a un sector muy especializado de la botánica, pero los pobladores de las zonas altoandinas donde crece la planta y dedicados a la medicina natural no tendrían problema alguno en reconocerla. Entonces, para las personas ajenas a la cultura andina no sería tan fácil reconocer a la planta elegida por Bustamante Vernal; por lo tanto, ella tuvo acceso al conocimiento de las tradiciones de los pueblos del Ande.

Según la investigadora Doris Walter, la planta Werneria nubigenea es conocida con el nombre común de "cebolla del abuelito", que tiene propiedades "medicinales" y se le debe utilizar de manera racional. Explica la razón de la siguiente manera:

No hay que recogerla de manera excesiva, si no el abuelito - antiguo dios del relámpago y del trueno en los tiempos prehispánicosse amarga, y manda una fuerte granizada de manera localizada en las chacras. Lo que significa destrucción de las cosechas, hambre y por consiguiente la muerte. (Walter, 2017, p. 158)

En la cultura andina los conocimientos ancestrales y tradicionales sobre el uso de las plantas medicinales en el Perú se mantienen vigentes hasta la actualidad, gracias al trabajo de los vendedores de plantas curativas que conocen del tema. Como los estudios sobre etnobotánica son escasos en el país, existe mucha confusión para la identificación de las plantas medicinales, porque según su lugar de origen se le asigna un nombre determinado, pero en otra localidad tiene un nombre diferente.

El dibujo representa a la planta medicinal Werneria nubígenea que no se menciona en la novela pero que alude a las tradiciones de los pueblos andinos que conservan sus conocimientos ancestrales a través de sus “depositarios" (Verde Márquez, 2016, p. 107): los curanderos y vendedores. La planta representa "un fin en sí mismo desde un punto de vista artístico", debido a que es el símbolo de "un pasado cultural valioso depositado en ella pero sin dejar de lado el devenir del tiempo" (Verde Márquez, 2016, pp. 114-115). En la ilustración "hay una fusión entre la idea del pasado cultural y los herederos de los creadores de dichas tradiciones" (Verde Márquez, 2016, pp. 99-100).

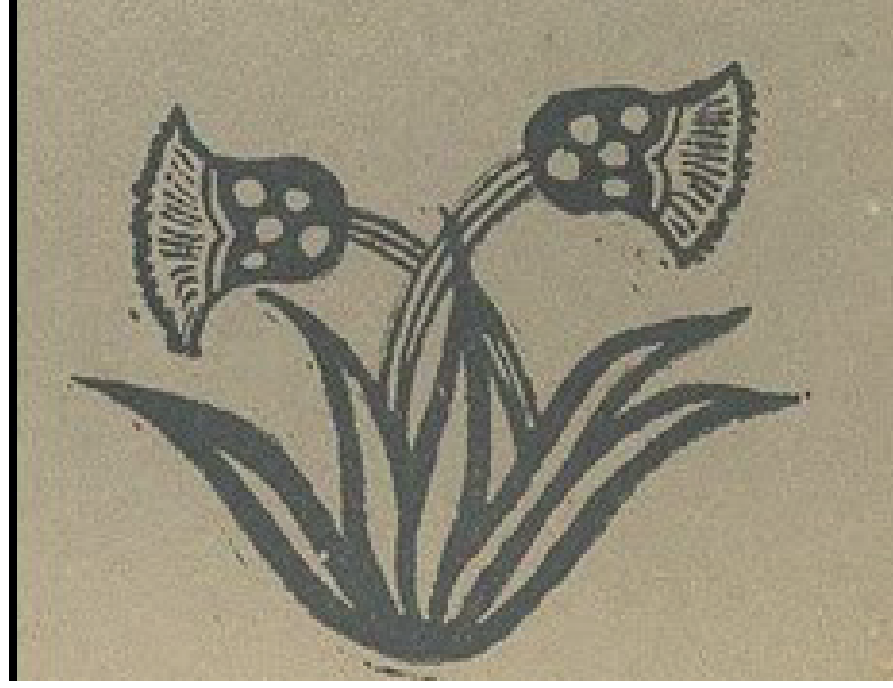

Figura 4.

Ilustración de Alicia Bustamante en Yawar fiesta (Arguedas, 1941, p. 164). Fuente: Biblioteca Nacional del Perú, 2020.

La tela cuadrangular de color marrón oscuro representa la enjalma. La corrida de toros fue traída por los españoles durante el virreinato, pero con el pasar del tiempo se convirtió en una tradición de los pueblos andinos en sus variadas celebraciones de fechas importantes. El sincretismo se concretó con la imposición de la religión católica y su adaptación a las creencias religiosas del Perú Antiguo.

Arguedas manifiesta en Yawar Fiesta (1941) que las telas llamadas enjalmas se cosen en el lomo del toro y le agregan objetos de valor; luego, en la ceremonia, las personas tratan de obtenerlas en la corrida. En el texto dice:

Las enjalmas las regalan las señoritas principales de nuestro pueblo; son enjalmas de seda, con monedas de plata y a veces de oro, en las puntas y en el bordado [...]. Al toro bravo se le cose la enjalma en el lomo, comenzando del morillo. Por la enjalma los indios se alocan, entran por tropas para arrancar la enjalma. (p. 38)

Bustamante Vernal para su carátula combinó el conocimiento tradicional que se mantiene en los pueblos del Ande, junto a las modificaciones inevitables en las costumbres aludido en la superficie cuadrangular de la enjalma. Bustamante Vernal dibujó a la planta Werneria nubigenea que representa a la flora nativa, que permanece estática, firme en la tierra, para indicar la vinculación de los pobladores con sus costumbres ancestrales. Mientras que la superficie cuadrangular detrás de la planta es el símbolo de la 
enjalma. La enjalma tiene los contornos ondulantes por el viento, es parte de una tradición traída por los españoles y tomada por los pueblos andinos en sus celebraciones, tiene las líneas "curvas" de la tela para dar la idea de "movimiento" (Morriña, 1982, p. 52). La enjalma representa el cambio en las tradiciones por las influencias foráneas que son asimiladas a través del tiempo.

\subsection{La agonía de Rasu Nĩiti ${ }^{\top}$ (1962)}

Este cuento escrito por Arguedas narra los últimos instantes de vida de "Pedro Huancaire", quien era un dansak" llamado Rasu-Ñiti que se vistió con su traje oficial para bailar por última vez, al compás de la música del arpista "Lurucha", junto al violinista "don Pascual", mientras su discípulo Atok' sayku ${ }^{9}$ observaba la escena (Arguedas, 1962, p. 15). Según el crítico literario Antonio Cornejo Polar (1973), La agonía de Rasu Niti es "un cuento admirable, el más bello de todos los que escribiera Arguedas" (p. 182).

Bustamante Vernal tomó de modelo un "mate burilado de Ayacucho" del año 1848, para ilustrar la carátula del libro La agonía de Rasu Niti (Arguedas, 1962, p. 4). La tapa del libro es de color marrón claro y lleva una tipografía negra. Las letras están dispuestas en un renglón en el margen superior en letras mayúsculas con el nombre del autor "JOSÉ MARÍA ARGUEDAS", y debajo el título del libro "LA AGONÍA DE 'RASU ÑITI"; en el tercer renglón está escrito el nombre de la editorial "camino del hombre" (véase imagen 5). La parte central de la carátula está conformada por una superficie rectangular de color marrón oscuro, que a su vez encierra a otra superficie rectangular, pero con sus cuatro esquinas redondeadas. En el interior están dibujados tres hombres, uno con sombrero, descalzo, de traje adornado de blondas, un pañuelo en la mano izquierda, y una tijera en la mano derecha; junto a otros dos hombres de sombrero en forma de copa, que tocan uno el arpa y el otro un violín rodeados de flores y hojas de una planta que decora la escena.

Luego de la descripción de los diseños hace falta identificarlos y explicar por qué Bustamante Vernal los eligió. En la ilustración, la flora está

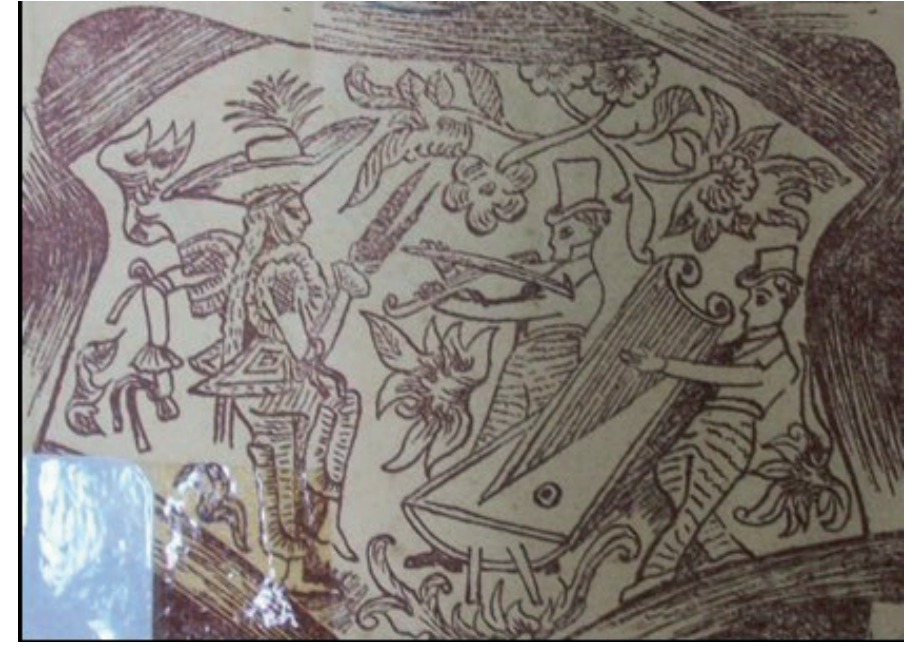

Figura 5.

Ilustración de Alicia Bustamante en La agonía de Rasu Niti (Arguedas, 1962, p. 1). Foto: Georgina Verde Márquez, 2015.

conformada por una planta conocida por el nombre de mate que corresponde a la especie Lagenaria vulgaris. Los diseños en los mates se realizan con la ayuda de un buril. La oca es una planta herbácea que decora el interior del mate, pertenece a la familia Oxalidacea y al género Oxalis tuberosa. Bustamante Vernal en el mate dibujó la representación de la danza de las tijeras, que consiste en complejas coreografías de un bailarín con una tijera en la mano, acompañado de un violinista y un arpista. Además, el tema del danzante de tijeras en la obra de Arguedas había sido mencionado con anterioridad en Tawar fiesta (1941, p. 5), también ilustrado por Bustamante Vernal. El mate de referencia que ella utilizó para el diseño de la carátula pertenece a la "Colección del Museo de la Cultura Peruana” (Jiménez Borja, 1948, p. 68). Cabe resaltar, que Bustamante Vernal tenía un amplio conocimiento del arte popular, debido a su afición de coleccionista, y a su trabajo en el Instituto de Arte Peruano, por tal motivo hizo una copia fiel de la imagen original del mate.

\subsection{Noticia histórica del actual edificio de la Universidad de San Marcos (1951)}

La Universidad Nacional Mayor de San Marcos fue creada el 12 de mayo del año 1551. Para la celebración del IV Centenario de su fundación en 1951 se publicó el libro El actual edificio de la Universidad Nacional Mayor de San Marcos. En dicho texto, el historiador Daniel Valcárcel 
escribió la primera parte titulada "Noticia histórica del actual edificio de la Universidad de San Marcos".

En el citado texto, Bustamante Vernal realizó tres dibujos a tinta china, cada uno a página completa que se detallan a continuación:

1.- "Lámina III. Patio de los Maestros (Facultades de Derecho y Ciencias Económicas) (Dibujo a Tinta China de AB)" (Valcárcel, 1951, p. 26). Bustamante Vernal dibujó un tramo de la arquitectura de un patio cuadrangular, compuesto por un corredor con columnas que sostienen arcos de medio punto las cuales rematan en un techo recto. La parte central del dibujo está conformada por un amplio jardín con una pileta.

2.-"Lámina IV. Patio de los Fazmines (Facultad de Letras) (Dibujo a Tinta China de AB)" (Valcárcel, 1951, p. 27). Bustamante Vernal dibujó el interior de uno de los corredores (véase imagen 6) de un patio cuadrangular, enmarcado por dos columnas con sus respectivos arcos de medio punto que dan paso a un amplio jardín, rodeado de arbustos de jazmines con una pileta en el centro.

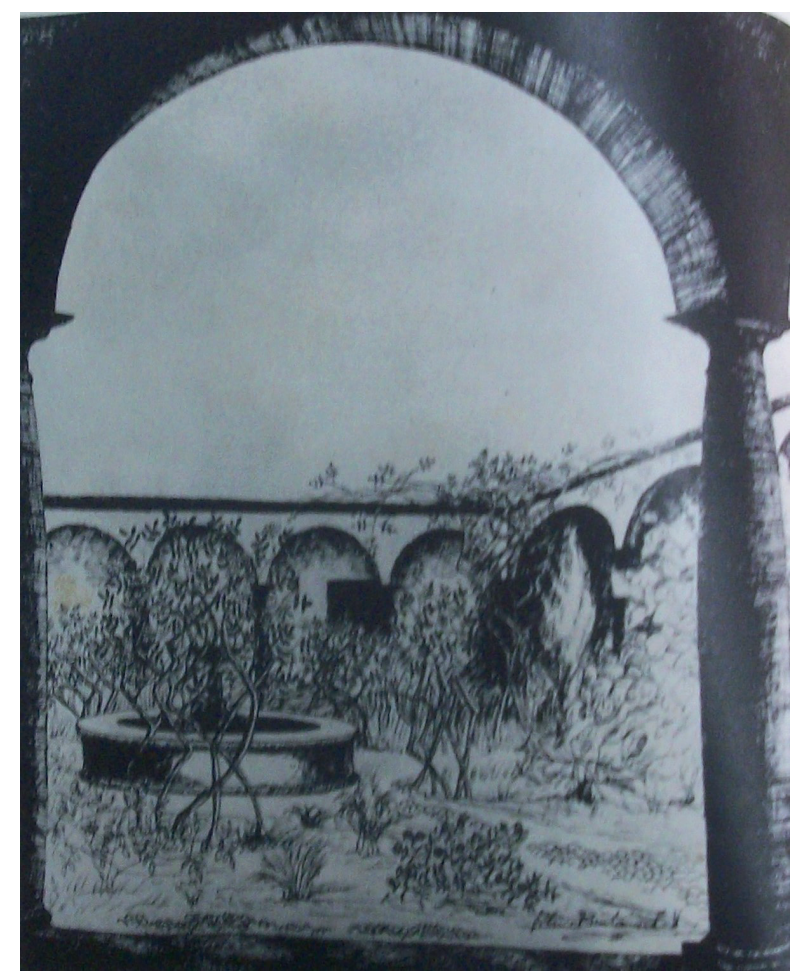

Figura 6

Patio de los Jazmines dibujado por Alicia Bustamante. Foto: Georgina Verde Márquez, 2019

3.-"Lámina V. Patio de los Naranjos (Facultad de Letras) (Dibujo a Tinta China de AB)" (Valcárcel, 1951, p. 30). Bustamante Vernal plasmó una parte

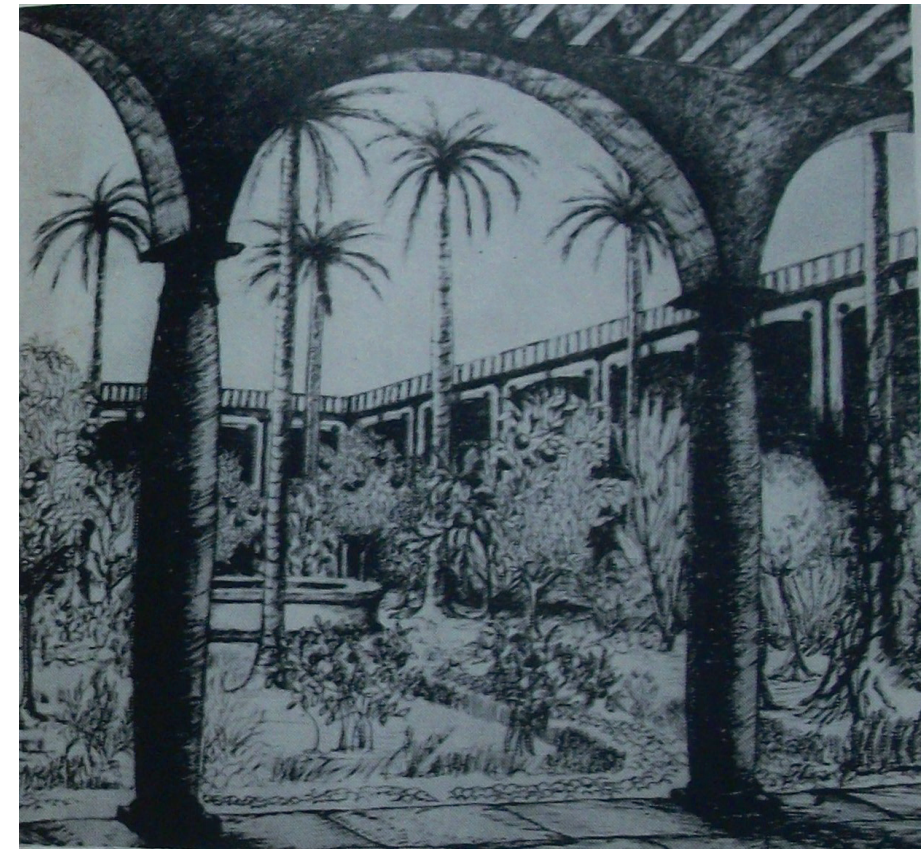

Figura 7

Patio de los Naranjos dibujado por Alicia Bustamante. Foto: Georgina Verde Márquez, 2019

de la arquitectura (véase imagen 7) de un corredor cuadrangular, conformado por columnas que sostienen arcos de medio punto las cuales remataban en un techo recto, además el jardín está rodeado por árboles de naranjos y algunas palmeras.

En el año de 1951 la Universidad Nacional Mayor de San Marcos funcionaba en el Convictorio de San Carlos, desde "el gobierno de Manuel Pardo (1872-1876)" (Valcárcel, 1951, p. 6). Del citado local, Bustamante Vernal consideró en sus dibujos la arquitectura y jardines de cada uno de los tres patios mencionados. El dibujo daba a conocer al público el recinto donde se impartían las clases para unirse a las celebraciones por los cuatrocientos años de antigüedad de la universidad.

\subsection{Revista 3}

Alicia Bustamante Vernal realizó cinco ilustraciones para el número 9 de la revista 3, que correspondía a los meses de septiembre-diciembre del año 1941. La primera viñeta está bajo el título de "VIÑETAS DE ALICIA BUSTAMANTE", la cual tiene a dos músicos (véase imagen 8), uno con sombrero que toca una tuba mientras un ave se acerca a la campana del instrumento; el otro con un gorro de militar toca una trompeta (Hernández, 1941, p. 4). La segunda viñeta es de dos burros dispuestos de perfil que se 
miran frente a frente, y en cuyas espaldas llevan una línea curva en forma de la letra "u", dentro de ella tiene una rama horizontal con hojas laterales (imparapinnada) (Mishkin, 1941, p. 6). La tercera viñeta corresponde a las siluetas de dos pavorreales de perfil, ambos frente a frente separados en medio por una pequeña flor (Xammar, 1941, p. 47), en el artículo llamado "Virgilio y Juan de Arona" de Luis Fabio Xammar que trata sobre el escritor Juan de Arona (Pedro Paz Soldán y Unanue), quien tradujo el primer libro de "Las Geórgicas de Virgilio" (1867) (Xammar, 1941, p. 43).

También en el texto se menciona al escritor José Arnaldo Márquez, pues se encargó de "satirizar a Juan de Arona y su traducción de las Geórgicas" (Xammar, 1941, p. 45). La cuarta viñeta es el dibujo de un camélido sudamericano que come las hierbas del suelo acompañado en el paisaje por una planta (Mejía, 1941, p. 70), en el artículo titulado "Relato en el tambo" por José Mejía Baca, que era un capítulo de la novela Sambambé, en el cual se describía el Tambo, un lugar de reposo para los viajeros (Mejía, 1941, p. 65). La quinta viñeta también está compuesta por dos burros dispuestos de perfil; el dibujo ilustra el artículo titulado "Significado de don Ricardo Palma en nuestra cultura" de Walter J. Peñaloza, que presentaba la trayectoria del escritor (Peñaloza, 1941, p. 94).

En el número 9 de la revista 3, Bustamante Vernal realizó los dibujos en parejas. El primer dibujo muestra un tema frecuente en la cerámica típica de la zona de Quinua, en el departamento de Ayacucho, que está compuesto por los músicos, uno vestido de civil y el otro de militar, así destaca el arte popular de los pueblos andinos. La segunda corresponde a la imagen de dos burros que representan el trabajo en una comunidad andina. La tercera está compuesta por dos pavorreales, debido al comportamiento ostentoso del ave cuando luce su plumaje, para compararlo con la vanidad de Arona, por su traducción de las Geórgicas de Virgilio, de la misma manera, a la actitud de Márquez por criticar al citado escritor. La cuarta viñeta es el dibujo de una llama que come las hierbas del suelo acompañada en el paisaje por un cactus (Mejía, 1941, p. 70). La llama se eligió "para resaltar la importancia que tiene en la economía" de las comunidades andinas (Verde Márquez, 2015b, p. 131). Además, el cactus es una planta xerófita. La llama descansaba en medio del paisaje, al igual que los viajeros cuando buscan reposo en el camino, y llegaban a la posada. La quinta viñeta consta de dos burros como elemento decorativo para el artículo sobre Ricardo Palma.

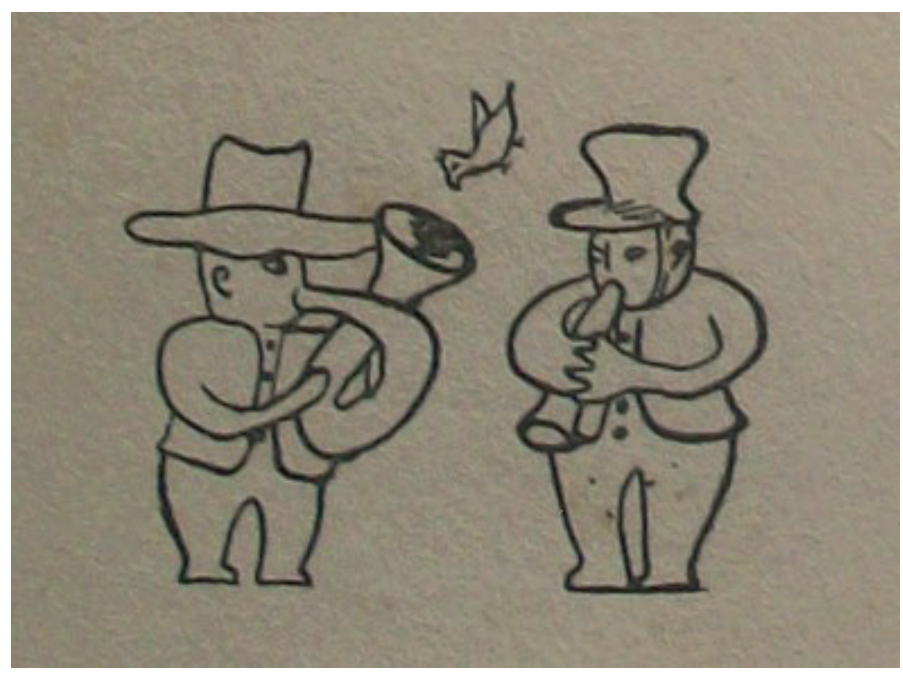

Figura 8

Viñeta de Alicia Bustamante (Hernández, 1941, p. 4). Foto: Georgina Verde Márquez, 2019.

José Alfredo Hernández había encomendado a Codesido la ilustración de Mitos y leyendas del Perú antiguo (1941) que formaba parte de las ediciones de la revista 3 (Verde Márquez, 2016, p. 66). Por lo tanto, la revista recurrió a ambas artistas para que colaboren con sus ilustraciones.

\subsection{El zumbayllü (1951)}

Arguedas (1951) escribió El zumbayllu que corresponde a un "fragmento inédito del capítulo sexto" de Los ríos profundos, publicado en el número 4 de la revista Letras Peruanas (p. 29). La narración iniciaba con la definición de la "terminación quechua illu", la cual representaba una "onomatopeya" de "la música que producen las pequeñas alas en vuelo, música inexplicable que surge del movimiento de objetos leves" (Arguedas, 1951, p. 4). También relacionó esa terminación con la descripción de un insecto llamado "tankayllu", conocido como "tábano zumbador" (Arguedas, 1951, p. 4). La siguiente escena transcurre en el patio del colegio donde causó un particular interés por parte del alumnado, el trompo llamado 
"zumbayllu", el cual fue llevado por Antero, cuyo sonido parecía "un coro de grandes tankayllus fijos en un sitio" (Arguedas, 1951, p. 5). El narrador menciona en el texto que cursaba el cuarto año de primaria, y su compañero Antero "estudiaba en el primer año de media; me adelantaba en dos grados" (Arguedas, 1951, p. 29).

Bustamante Vernal realizó la "ilustración" de El zumbayllu (Arguedas, 1951, p. 4). En el dibujo utilizó el color rojo violáceo. Una flor en forma de campana está (véase imagen 9) ubicada en el ángulo inferior izquierdo desde el punto de vista del espectador y tiene frente a ella a sus tres inflorescencias en forma de capullo. La flor y los tres capullos están unidas por un solo tallo a manera de una enredadera, también cada capullo tiene una hoja a su lado. En el margen superior figura un caballo de perfil con las patas delanteras y traseras algo extendidas. El caballo tiene el pelaje del cuello en posición horizontal, y la cola de forma ondulante. Las letras iniciales del nombre de la artista $\mathrm{ABV}$ se observan en el margen inferior derecho desde el punto de vista del espectador.

Luego de la descripción de los diseños hace falta identificarlos y explicar por qué Bustamante Vernal los eligió. Después de varios años de investigación se logró identificar a la planta que tiene una apariencia blanquecina con pelos que la recubren, posee flores amarillas encerradas en la cabezuela. Su nombre científico es Culcitium canescens. No fue fácil dar con el nombre de la planta, pues existen muchas especies dentro de la familia de las Asteráceas. Además, tal dato es poco conocido hasta para los especialistas en etnobotánica dentro del ámbito académico. En cambio, los vendedores de plantas medicinales de la zona andina donde crece la planta no tendrían problema en reconocerla por sus propiedades curativas. Bustamante Vernal tenía muchos referentes sobre las tradiciones de los pueblos del Ande por su contacto con los artistas populares, quienes compartían con ella sus conocimientos.

La planta, por sus inflorescencias cerradas en forma de campana, junto a un brote en flor, indica dos estadios diferentes de crecimiento. Por citar un ejemplo, las flores en el arte llevan un mensaje implícito debido a que "en capullo corresponden a la juventud [...] y las flores con sus hojas expresan que ya está entrando a otra etapa de su vida" (Verde Márquez, 2016, p. 149). Se puede hacer extensivo

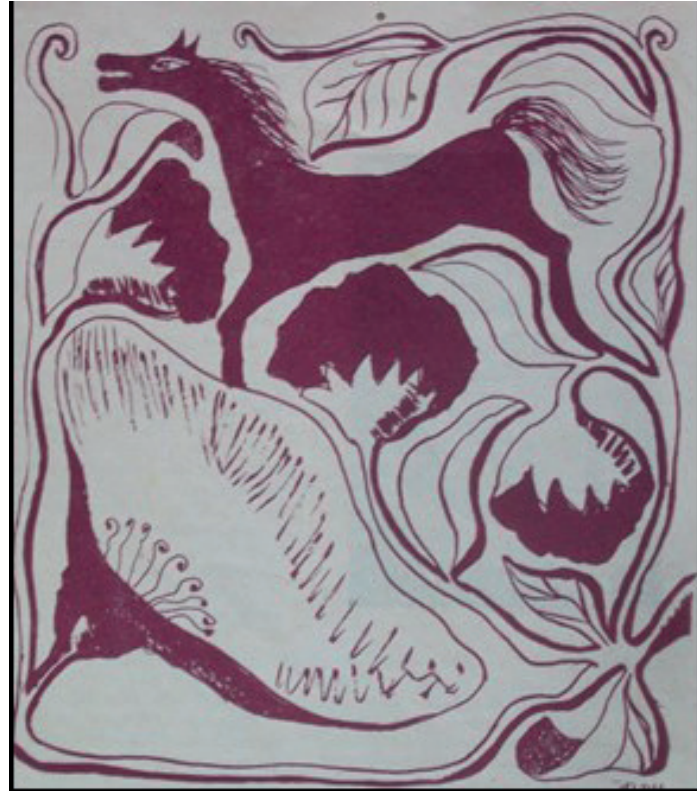

Figura 9 Ilustración de Alicia Bustamante (Arguedas, 1951, p. 4). Foto: Georgina Verde Márquez, 2019.

el análisis de un cuadro pintado por Julia Codesido a la ilustración de Bustamante Vernal, a través del siguiente texto:

También trae a colación el paso del tiempo que rige a todos los seres vivos, hecho evidente por los diferentes estadios de las flores dispuestas [...]. Debido a que todos los seres vivos atraviesan varias etapas de desarrollo, se puede hacer una comparación entre la vida de una flor con los años de vida de una persona. (Verde Márquez, 2016, p. 150)

El narrador del texto tenía aproximadamente nueve años, y su compañero Antero doce; por tal razón, el menor cursaba la primaria y el mayor la secundaria (Arguedas, 1951, p. 29). Se puede inferir que las plantas representan las edades de ambos personajes de la historia, precisamente por el tema de la novela sobre ellos en su vida escolar, que pasan de la niñez a la adolescencia. El caballo tiene las patas algo extendidas y flexionadas, lo que indica la idea de movimiento. Esta forma de representación en el arte fue empleada en la obra Desnudo bajando una escalera (1912) de Marcel Duchamp (1887-1968), donde las piernas del personaje están flexionadas y reproducidas en varios planos para crear el dinamismo. Además, la crin en línea horizontal es semejante a la forma que adquiere el pelaje cuando los caballos corren y el viento levanta su pelaje. El pelaje de la cola también está desordenado por la acción del viento. El color rojo está asociado a la idea de "fuerza" y "vitalidad" 
(Morriña, 1982, p. 89), y en el dibujo el caballo se relaciona con la libertad y vitalidad propia de la niñez, debido a la edad de los protagonistas de la historia. Debe considerarse que el caballo pequeño tiene un temperamento indómito que necesita de un entrenador para ser amaestrado. Los protagonistas de la historia reciben una educación en la escuela, allí se interrelacionan para adaptarse a la convivencia en grupo. En el patio del colegio se reúnen por la curiosidad que despierta en ellos el juego de hacer bailar al Zumbayllu.

En la ilustración, Bustamante Vernal plasmó una vez más la naturaleza: la flora representada por la planta medicinal Culcitium canescens y la fauna por el caballo.

\subsection{Orovilca ${ }^{11}(1954)$}

Arguedas publicó Orovilca en el número 11 de la revista Letras Peruanas (1954, p. 45). El texto trata sobre Salcedo, un estudiante destacado de un colegio quien se "bañaba" en la laguna Orovilca, la cual tenía una "arenilla dorada" (Arguedas, 1954, p. 48). Él creía en la historia de "una corvina de oro" que viajaba entre el "mar" y la "laguna", e iba "nadando sobre las dunas" (Arguedas, 1954, p. 49). Salcedo tenía un compañero llamado "Wilster que lo odiaba" y también lo "acosaba" (Arguedas, 1954, p. 45); ambos pelean, pero Salcedo terminó ensangrentado, y decidió irse del colegio (Arguedas, 1954, p. 49).

La ilustración de Orovilca realizada por Bustamante Vernal consta de un jardín (véase imagen 10) donde crece un árbol de tronco delgado que en lo alto de sus ramas sostiene a un ave (Arguedas, 1954, p. 45). Detrás de la planta está el pórtico compuesto por dos arcos de medio punto, los cuales dan paso al corredor del recinto. En el vano del arco de medio punto de la izquierda figura la silueta de un joven de perfil, con su espalda y pie apoyados en el muro mientras leía un libro.

Luego de la descripción de la ilustración hace falta explicar su significado. Según el texto, el árbol de la ilustración es del género Ficus, y el ave un chaucato (Mimus longicaudatus), porque en un párrafo decía "sobre uno de los grandes ficus que dan sombra al claustro del Colegio [sic], cantó un chaucato" (Arguedas, 1954, p. 45). Además, la silueta del joven apoyado en el lado izquierdo del vano del arco de medio punto representa a Salcedo. En la historia se menciona que él "leía, mientras paseaba" en el colegio (Arguedas, 1954, p. 45).

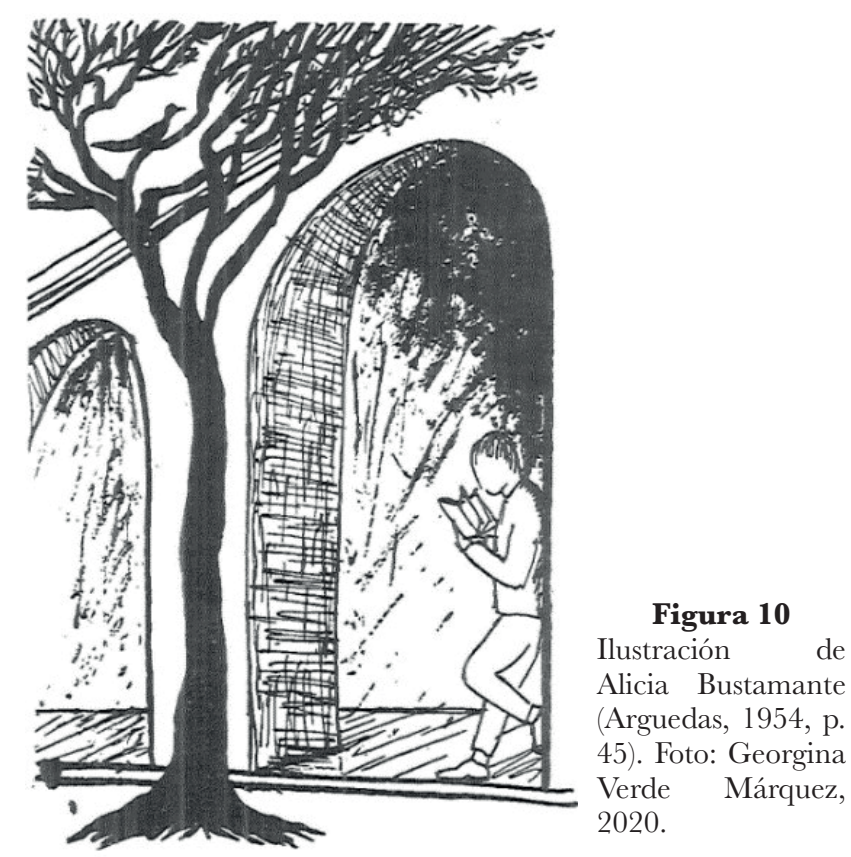

Bustamante Vernal ilustró la naturaleza. La flora está presente en el árbol llamado ficus, la fauna en el ave conocida como chaucato. Ella dibujó en el fondo la fachada interior del claustro con sus arcos de medio punto, de esta manera plasmó la arquitectura del recinto que corresponde al espacio donde los alumnos con frecuencia transitaban.

\section{Conclusiones}

- Bustamante Vernal siempre ejerció su labor de artista plástica a lo largo de su vida. Se comprueba con su trabajo en la ilustración de textos, y en la presentación de sus dos exposiciones individuales, la primera en 1944 y la segunda en 1955.

- Bustamante Vernal, desde 1938 hasta 1962, colaboró con Arguedas en tres carátulas: primero en Canto kechwa (1938), luego en Yawar fiesta (1941), y por último en La agonía de Rasu Ñiti (1962); también en dos cuentos: $E l$ zumbayllu (1951) y Orovilca (1954). Ellos tenían un vínculo familiar y un mutuo interés por el estudio de la cultura peruana. 
- Bustamante Vernal ilustró los libros Canto kechwa (1938) y Yawar fiesta (1941) de Arguedas usando elementos de la naturaleza. En el primero de ellos está presente la flora mediante la planta de quinua (Chenopodium quinoa), y la fauna representada por la vicuña (Vicugna vicugna mensalis). También la naturaleza, de igual manera, está presente en las viñetas interiores que representan a una planta de maguey, peces y aves. En el segundo libro, ella dibujó la planta medicinal Werneria nubigenea para evidenciar los conocimientos tradicionales que conservan los habitantes andinos, junto a las nuevas costumbres aludidas en la tela de la enjalma que simboliza el mestizaje cultural.

- En el libro titulado La agonía de Rasu Ñiti (1962), escrito por Arguedas, la ilustración de la carátula por Bustamante Vernal es la representación de la tradición del burilado de mates, creado por los artistas populares, con la escenificación de la danza de las tijeras que corresponde a la temática del libro. Además, la flora está presente en la planta Lagenaria vulgaris que es el mate en sí, y la oca (Oxalis tuberosa) en la decoración.

- El texto titulado "Noticia histórica del actual edificio de la Universidad de San Marcos" (1951), que conformaba el libro El actual edificio de la Universidad Nacional Mayor de San Marcos escrito por Daniel Valcárcel, contiene los dibujos de Bustamante Vernal que ilustran los tres patios de la Casona con sus jardines y respectiva arquitectura, primero el Patio de los Maestros (Facultades de Derecho y Ciencias Económicas), el Patio de los fazmines, y por último el Patio de los Naranjos (Facultad de Letras). Con ello se unía a las celebraciones por los cuatrocientos años de fundación de la citada casa de estudios.

- Bustamante Vernal realizó cinco ilustraciones para el número 9 de la revista 3 en 1941. Primero ilustró a dos músicos, un tema del arte popular frecuente en la cerámica de Quinua, en el departamento de Ayacucho. La segunda trata de dos burros que representan el trabajo en una comunidad andina. La tercera está compuesta de dos pavorreales que expresan la vanidad de Juan de Arona (Pedro Paz Soldán y Ugarte), y José Arnaldo Márquez. La cuarta corresponde a una llama junto a un cactus, para aludir a la búsqueda de alimento en una posada que realizan los viajeros. La quinta representa a dos burros, como elemento decorativo. Las viñetas tratan sobre el arte popular, la fauna y la flora.

- Bustamante Vernal, en la revista Letras Peruanas, ilustró El zumbayllu (1951), y Orovilca (1954), ambos textos de Arguedas. Primero se basó en la naturaleza plasmando la planta Culcitium canescens, que presenta dos estadios (en forma de capullo y en flor), para aludir las etapas de crecimiento de los alumnos de un colegio. Luego, la fauna presente por el caballo, que expresa la libertad propia de la niñez. En el segundo caso, en su dibujo une a la arquitectura del recinto al protagonista de la historia, que es el alumno Salcedo. También la naturaleza está presente en el dibujo: la flora en el árbol del género de los Ficus, y la fauna por el ave llamada chaucato (Mimus longicaudatus).

\section{NOTAS}

1 Existe una confusión que se mantiene hasta la actualidad sobre esta exposición, al considerar que se realizó en "1956 (?) [...] en la Galería Lima" (Zevallos de Vasi, 1974, p. 15). Sin embargo, la segunda exposición fue en el año de 1955, en el Instituto de Arte Contemporáneo, como se ha precisado.

2 Expreso mi profunda gratitud y agradecimiento a la familia Bustamante por permitir la publicación de las imágenes que ilustran el presente artículo.

3 Alejandro Ortiz refiere en el texto la dificultad para conocer los nombres científicos de la flora y fauna mencionadas en las canciones; por ejemplo: la planta llamada "nuchku" de la canción Raki-raki no fue identificada ni siquiera por los habitantes de la zona (Ortiz, 1997, p. 362). 
4 La palabra yawar proviene del quechua, traducida al español significa "sangre" (Arguedas, 1958, p. 157).

5 La palabra del idioma quechua turupukllay, es el resultado de "toro y de pukllay, jugar, jugada de toros" (Arguedas, 1958, p. 159).

6 El auki K'arwarasu tiene tres picos de nieve, "es el padre de todas las montañas de Lucanas" (Arguedas, 1958, p. 104).

7 Rasu Niti es una frase quechua cuya traducción al español significa el "quien aplasta la nieve" (Arguedas, 1962, p. 8).

8 Dansak' procede del idioma quechua, cuya traducción al español es "bailarín" (Arguedas, 1962, p. 8).

9 Atok' sayku se refiere a una palabra quechua que traducida al español significa "cansa al zorro" (Arguedas, 1962, p. 15).

10 Zumbayllu es una palabra del idioma quechua, que en español significa "trompo" (Arguedas, 1951, p. 5).

11 Orovilca proviene del idioma quechua, cuya traducción al idioma español significa "gusano sagrado" (Arguedas, 1954, p. 45). Este texto fue obtenido gracias al archivo del Centro de Estudios Literarios Antonio Cornejo Polar.

\section{Referencias bibliográficas}

Arguedas, J. (1954). Orovilca. Letras Peruanas, 11(45), 48-49.

Arguedas, J. (1938). Canto kechwa. Ediciones "Club del Libro Peruano".

Arguedas, J. (1941). Yawar fiesta. C.I.P. http://bibliotecadigital.bnp.gob.pe/portal-bnpweb/\# /libro/LIB-365

Arguedas, J. (1951). El zumbayllo. Letras Peruanas, 4(4-5), 29.

Arguedas, J. (1958). Yawar fiesta. Editorial Juan Mejía Baca, Ediciones Populares.

Arguedas, J. (1962). La agonía de Rasu Niti. Ediciones "Camino del hombre".

Carpio, K. \& Yllia, M. E. (2006). Alicia y Celia Bustamante, la Peña Pancho Fierro y el Arte Popular. Illapa, 3, 45-60.

Castrillón, A. (2001). Los independientes. Instituto Cultural Peruano Norteamericano.

Cornejo Polar, A. (1973). Los universos narrativos de José María Arguedas. Editorial Losada.

Escuela Nacional de Bellas Artes. (1922). Monografía histórica y documentada sobre la Escuela Nacional de Bellas Artes desde su fundación hasta la segunda exposición oficial. Escuela Nacional de Bellas Artes.

Exposición en el IAC. (1955, 23 de octubre). En El Comercio, 17.

Falcón, J. (1989). Enrique Camino Brent. Instituto Sabogal de Arte.

Fernández, J. (1972). Antología de la Independencia. Comisión Nacional del Sesquicentenario de la Independencia del Perú.

Fuentes, M. (1944, 6 de noviembre). La exposición pictórica de Alicia Bustamante. En La Crónica, 3.

Gesualdo, V. (1968). Enciclopedia del arte en América, Biografías III. Buenos Aires: Bibliográfica OMEBA.

Gonzáles, D. (2005). 50 años del voto femenino en el Perú: historia y realidad actual. Lima: Ministerio de la Mujer y Desarrollo Social.

Hernández, J. (1941). Colaboran en este número, 3, 4.

Jiménez Borja, A. (1948). Mate peruano. Revista del Museo Nacional, 17, 34-74.

Martínez Compañón, B. (1998). Trujillo del Perú, vol. Il. Madrid: Ediciones Cultura Hispánica.

Mejía, J. (1941). Relato en el tambo, 3, 65-70.

Milla, C. (1996). Diccionario histórico y biográfico del Perú. Siglos XV-XX, vol. 3. Editorial Milla Batres.

Mishkin, B. (1941). Posesión de la tierra en una aldea, 3, 5-11. 
Morriña, O. (1982). Fundamentos de la forma. La Habana: Universidad de La Habana. Facultad de Artes y Letras. Departamento de Historia del Arte.

Ortiz, A. (1997). La metáfora de los amores salvajes en el "Canto kechwa" de José María Arguedas. Anthropologica, 15(15), 355-369. https://revistas.pucp.edu.pe/index.php/ anthropologica/article/view/1230

Peñaloza, W. (1941). Significado de don Ricardo Palma en nuestra cultura, 3, 73-95.

Rodríguez, J. (1984). Las variantes textuales de Yawar fiesta de José María Arguedas. Lexis, 8(1), 1-93. https://revistas.pucp.edu.pe/index.php/lexis/article/view/5068

Salazar Bondy, S. (1958, 21 de enero). Visita al salón del arte abstracto. En La Prensa, p. 8.

Tauro del Pino, A. (1966). Diccionario Enciclopédico del Perú llustrado, vol. II. Lima: Editorial Juan Mejía Baca.

Valcárcel, D. (1951). Noticia histórica del actual edificio de la Universidad de San Marcos. En D. Valcárcel y G. Ibscher (Eds.), El actual edifico de la Universidad Nacional Mayor de San Marcos (1-36). Lima: Universidad Nacional Mayor de San Marcos. Publicaciones del IV Centenario.

Verde Márquez, G. A. (2015a). El cóndor en la obra de Julia Codesido. (Tesis para optar por el grado de Licenciada en Arte). Universidad Nacional Mayor de San Marcos. Facultad de Letras y Ciencias Humanas, Lima, Perú. https://hdl.handle.net/20.500.12672/9263

Verde Márquez, G. A. (2015b). Ilustración de libros y revistas por Julia Codesido. Tesis, 8 (8), 125-136.

Verde Márquez, G. A. (2016). La representación femenina en la obra de Julia Codesido (19201938). (Tesis para optar por el grado de Magíster en Arte Peruano y Latinoamericano). Universidad Nacional Mayor de San Marcos. Facultad de Letras y Ciencias Humanas, Lima, Perú. https://hdl.handle.net/20.500.12672/8393

Walter, D. (2017). Algunos aportes a la etnobotánica en la Cordillera Blanca (Sierra de Ancash). Indiana, 34 (1), 149-176. https://dx.doi.org/10.18441/ind.v34i1.149-176.

Wiesse, M. (1957). José Sabogal, el artista y el hombre. Compañía de Impresión y Publicidad S.A.

Xammar, L. (1941). Virgilio y Juan de Arona, 3, (9), 42-47.

Zevallos de Vasi, R. (1974). Homenaje a Alicia y Celia Bustamante. En Cuadernos de Arte y de Historia, vol. 2 (12-19). Lima: Museo de Arte y de Historia y de la Sección de Arte del Departamento de Humanidades. 